\title{
Influence of Adjuvants on the Control of Glyphosate-Resistant Canada Fleabane and Waterhemp in Corn with Tolpyralate
}

\author{
Nicole M. Langdon', Nader Soltani1 ${ }^{*}$, Alan J. Raedar², David C. Hooker ${ }^{1}$, Darren E. Robinson1, \\ Peter H. Sikkema ${ }^{1}$
}

${ }^{1}$ University of Guelph Ridgetown Campus, Ridgetown, Canada

${ }^{2}$ ISK Biosciences Inc., Concord, OH, USA

Email: *soltanin@uoguelph.ca

How to cite this paper: Langdon, N.M., Soltani, N., Raedar, A.J., Hooker, D.C., Robinson, D.E. and Sikkema, P.H. (2020) Influence of Adjuvants on the Control of Glyphosate-Resistant Canada Fleabane and Waterhemp in Corn with Tolpyralate. American Journal of Plant Sciences, 11, 354-371. https://doi.org/10.4236/ajps.2020.113026

Received: February 13, 2020

Accepted: March 20, 2020

Published: March 23, 2020

Copyright $\odot 2020$ by author(s) and Scientific Research Publishing Inc. This work is licensed under the Creative Commons Attribution International License (CC BY 4.0).

http://creativecommons.org/licenses/by/4.0/

(c) (i) Open Access

\begin{abstract}
Tolpyralate is a new benzoylpyrazole herbicide for weed management in corn. It is recommended to be co-applied with atrazine along with the adjuvants methylated seed oil concentrate (MSO) plus an ammonium nitrogen fertilizer, such as urea ammonium nitrate (UAN). Two studies were conducted on glyphosate-resistant (GR) Canada fleabane and GR waterhemp to determine if an additional adjuvant is still required when tolpyralate plus atrazine are tankmixed with a commercial glyphosate formulation (Roundup WeatherMAX ${ }^{\circledast}$ ) in corn. Trials were conducted over a two-year period (2018-19) on farms in south western Ontario with confirmed GR populations. When co-applied with Roundup WeatherMAX ${ }^{\oplus}$, the addition of MSO to tolpyralate + atrazine increased control of GR waterhemp 9\%; however, there was no increase in GR Canada fleabane control from the addition of additional adjuvants. At $8 \mathrm{WAA}$, all treatments provided $>91 \%$ and $>84 \%$ control of GR waterhemp and GR Canada fleabane, respectively. This study concludes that the addition of Roundup Weathermax ${ }^{\circledast}$ to tolpyralate plus atrazine improves the control of GR waterhemp and GR Canada fleabane in corn.
\end{abstract}

\section{Keywords}

Biomass, Crop Injury, Density, Sensitivity, Weed Control, Yield, Amaranthus tuberculatus, Conyza canadensis L. Cronq

\section{Introduction}

Glyphosate-resistant (GR) waterhemp (Amaranthus tuberculatus) and GR Canada fleabane (Conyza canadensis L. Cronq.) are prevalent across parts of south- 
ern Ontario and negatively affect net returns for affected farmers. Waterhemp is a dioecious, competitive, troublesome weed with an extended emergence from early spring until October [1]. Early emerging waterhemp has a competitive advantage over later emerging summer annual weeds, and the extended emergence pattern necessitates season-long residual weed control programs for the control of late emerging cohorts [2] [3]. Following pollination, one female waterhemp plant can produce up to 4.8 million small, black seeds in ideal conditions [2]. Seeds can remain viable in the soil for up to two decades; however, most seeds will germinate or decay within the first three years [3] [4] [5]. Waterhemp seeds can be transported by human activity, equipment, via waterways and by migratory waterfowl. As a dioecious plant, waterhemp requires cross pollination, resulting in genetic and phenotypic variability, accelerated spread of resistance genes, and the ability to adapt to diverse environmental conditions [6]. Globally, there are waterhemp biotypes with resistance to seven herbicide modes of action: acetolactate synthase inhibitors, synthetic auxins, photosystem II inhibitors, 5-enolpyruvyl shikimate-3-phosphate synthase inhibitors (EPSPS), protoporphyrinogen IX oxidase inhibitors, very long chain fatty acid elongases inhibitors, and 4-hydroxyphenyl-pyruvate dioxygenase inhibitors (HPPD), belonging to WSSA Herbicide Groups 2, 4, 5, 9, 14, 15 and 27, respectively [7]. The first GR waterhemp population in Ontario was confirmed from seed collected in 2014 in Lambton county [1]. After the initial discovery, GR waterhemp is now present in 11 Ontario counties [1] [8], a distance of $500 \mathrm{~km}$. Waterhemp biotypes in Ontario have confirmed resistance to Groups 2, 5, 9, and 14 herbicides [7]. Of the waterhemp populations in Ontario, it was estimated that $61 \%$ have 3 -way multiple resistance to imazethapyr, atrazine and glyphosate [1]. Glyphosate resistance in waterhemp can be due to target or non-target site mechanisms. EPSPS gene amplification, an amino acid substitution (Pro-Ser) at position 106, and reduced translocation are herbicide resistance mechanisms that confer resistance to glyphosate [9] [10]. Compared to other Amaranthus species, waterhemp is not as competitive as Palmer amaranth (A. palmeri S. Wats); however, it is more competitive and causes greater yield reductions than redroot pigweed ( $A$. retroflexus L.) [11].

Canada fleabane is a widely adapted winter or summer annual weed that is native to North America [12]. The ability for Canada fleabane seeds to germinate in the fall and over-winter as rosettes allows plants to establish and grow without competition from neighbouring summer annual weeds and crops [12]. Seedlings that emerge in the spring will bypass the rosette stage and begin stem elongation soon after emergence [13]. In Ontario, Canada fleabane seed can germinate throughout the year if temperatures exceed the base germination temperature range of $8.0^{\circ} \mathrm{C}-9.5^{\circ} \mathrm{C}$; the extended emergence pattern requires residual herbicides for full-season weed control [14]. Canada fleabane is a self-pollinating species that begins flowering in mid-July and matures by mid-September [12]. Canada fleabane is a prolific seed producer, with seed production per plant proportional to plant height; a $1.5-\mathrm{m}$ tall plant can produce up to 230,000 seeds per 
plant [12] [15]. Canada fleabane seeds have a small attached pappus that aids in wind dispersal; seeds can travel long distances of up to $500 \mathrm{~km}$ [16]. Canada fleabane seeds germinate on or near the soil surface, at depths up to $0.5 \mathrm{~cm}$, making this species well-adapted to no-till crop production systems [17]. Multiple applications of glyphosate are often used for weed control in no-till GR cropping systems; this may have contributed to the evolution of GR Canada fleabane biotypes [17]. The first confirmed population of GR Canada fleabane was discovered in 2001 in a GR soybean field near Delaware, USA, that received glyphosate applications for three consecutive years [18]. In 2010, the first population of GR Canada fleabane was discovered in Ontario in Essex County, and by 2015, GR populations were found in 30 counties across southern Ontario from the Michigan to the Quebec border [19]. Resistance in GR Canada fleabane is primarily caused by non-target site resistance mechanisms: vacuolar sequestration and enhanced metabolism where the herbicide does not reach the target site at a lethal dose [20] [21]. More recently, a proline to serine amino acid substitution at position 106 was identified that confers resistance to glyphosate by altering the target site for herbicide binding [22]. Resistance to herbicides adds complexity to the management of Canada fleabane.

Tolpyralate is a Group 27, HPPD-inhibiting herbicide for use in corn that is recommended to be applied as a tankmix with atrazine [23] [24] [25] [26]. Tolpyralate plus atrazine, applied POST, provides control of annual grass and broadleaf weed species including Canada fleabane and Amaranthus spp. [24] [27]. On the Canadian and US labels, tolpyralate plus atrazine provides "control" of both common ( $A$. rudis) and tall ( $A$. tuberculatus) waterhemp; however, only the US label lists "partial control" of Canada fleabane [25] [28].

The recommended adjuvants with tolpyralate are MSO Concentrate $(1 \% \mathrm{v} / \mathrm{v})$ and a nitrogen source such as UAN or ammonium sulfate (AMS) $(2.5 \% \mathrm{v} / \mathrm{v})$ to improve herbicide efficacy [28]. Defined by the Weed Science Society of America, an adjuvant is any substance added to the spray tank, or present in an herbicide formulation that enhances herbicidal activity or application characteristics. Selection of an appropriate adjuvant at the proper concentration can improve herbicide coverage on various leaf surfaces [29]. Adjuvants can decrease surface tension and contact angle (angle between the leaf surface and the spray droplet), as well as influence wetted area and evaporation time, and play a role in the deposition patterns on leaf surfaces [30]. Many herbicides require the addition of an adjuvant for optimal performance. Topramezone, another Group 27 herbicide, has enhanced efficacy with the addition of MSO due to increased absorption and translocation of the herbicide in the plant [31]. Quinclorac efficacy is enhanced with the addition of MSO and a nitrogen fertilizer that combats antagonistic salts present in the carrier water [32].

Adjuvants can also be already present in the herbicide formulation. For example, most commercial glyphosate formulations in Canada are formulated with an adjuvant. For example, Roundup WeatherMAX ${ }^{\otimes}$ contains proprietary surfactants to improve the penetration of the herbicide into the plant tissue [33]. In a 
tankmix, glyphosate formulations have been found to influence crop injury [34], weed control [35] [36] or absorption and translocation of the tank mix partners. Adjuvants add an additional cost to the complete weed control program. In Ontario, adjuvants can cost up to $\$ 10.00 \mathrm{ha}^{-1}$ (Agris Co-operative, personal communication) and require the handling of multiple product containers.

Based on previous research, it is evident that there is an interaction between commercial glyphosate formulations and various herbicides that may be the result of the aggressive adjuvant system in the formulation. Roundup Ready ${ }^{\otimes}$ hybrids account for $96 \%$ of corn acreage in Eastern Canada (M. Reidy, Stratus, personal communication), thus the most common use pattern for tolpyralate plus atrazine will be tankmixed with glyphosate. It is unknown whether the adjuvant in Roundup WeatherMAX ${ }^{\circledast}$ is sufficient for maximum herbicide efficacy with tolpyralate plus atrazine, or if additional adjuvants are still required [33]. The objective of this study was to determine if additional adjuvants (MSO and UAN) are required when tolpyralate plus atrazine is tankmixed with Roundup WeatherMAX ${ }^{\circledast}$ for the control of GR waterhemp and GR Canada fleabane.

\section{Materials and Methods}

This manuscript summarizes two distinct studies: Study 1- Impact of adjuvants on tolpyralate + atrazine efficacy for the control of GR waterhemp, and Study 2Impact of adjuvants on tolpyralate + atrazine efficacy for the control of GR Canada fleabane.

\subsection{Study 1}

Six field trials (as listed in Table 1) were conducted over a two-year period $(2018,2019)$ at sites with confirmed GR waterhemp on Walpole Island and near Cottam, ON [1]. The seedbed was prepared with two passes of a cultivator with rolling basket harrows. All sites were fertilized according to soil test results and crop requirements; the fertilizer was applied prior to seeding and incorporated into the soil. DKC45 - 65 RIB corn (Monsanto Co., St. Louis, MO) was seeded at approximately 83,000 seeds $\cdot \mathrm{ha}^{-1}$ to a depth of $4 \mathrm{~cm}$ in rows spaced $75 \mathrm{~cm}$ apart. The experimental design was a two-way, 2 by 5 factorial: Factor One was Roundup WeatherMAX ${ }^{\oplus}$, and Factor Two was five tolpyralate + atrazine treatments including: no tank mix partner, tolpyralate + atrazine, tolpyralate + atrazine + MSO, tolpyralate + atrazine $+\mathrm{UAN}$, and tolpyralate + atrazine + MSO + UAN. Methylated seed oil (MSO Concentrate ${ }^{\oplus}$, Loveland Products Inc., Loveland (O) and 28\% UAN were added according to label directions. Trials were organized as a randomized complete block with 4 replicates. Plots were $2.25 \mathrm{~m}$ wide ( 3 corn rows spaced $75 \mathrm{~cm}$ apart) and $8 \mathrm{~m}$ long. To remove susceptible waterhemp biotypes and non-target weed species, glyphosate ( $\left.450 \mathrm{~g} \cdot \mathrm{ae} \cdot \mathrm{ha} \mathrm{a}^{-1}\right)$ was applied after crop emergence using a tractor mounted sprayer to the entire experimental area. Table 1 lists waterhemp sites, planting and herbicide application dates. 
Table 1. Year, location, planting and herbicide application dates for six trials on control of glyphosate-resistant waterhemp with Roundup WeatherMax ${ }^{\otimes}$ plus tolpyralate plus atrazine plus adjuvants, applied postemergence, in Ontario in 2018 and 2019.

\begin{tabular}{ccccc}
\hline Species & Year & Nearest Town & Planting Date & Herbicide Application Date \\
\hline GR Waterhemp & 2018 & Cottam & June 2 & June 21 \\
& 2018 & Walpole Island & June 1 & June 29 \\
2018 & Walpole Island & June 11 & July 9 \\
2019 & Cottam & June 19 & July 10 \\
2019 & Walpole Island & June 25 & July 16 \\
2019 & Walpole Island & June 25 & July 18 \\
\hline
\end{tabular}

Herbicide treatments were applied with a $\mathrm{CO}_{2}$ pressurized backpack sprayer calibrated to deliver $200 \mathrm{~L} \cdot \mathrm{ha}^{-1}$ at $240 \mathrm{kPa}$ equipped with a $1.5 \mathrm{~m}$ handheld boom. The boom was equipped with four ULD12002 nozzles (Pentair, New Brighton, MN, USA) spaced $50 \mathrm{~cm}$ apart producing a spray width of $2 \mathrm{~m}$. Herbicides were applied when GR waterhemp averaged $10 \mathrm{~cm}$ in height.

Corn injury at 1,2 and 4 WAA, and weed control at 2, 4, 8 and 12 WAA was assessed visually on a percent scale where 0 indicates no corn injury/weed control and 100 complete plant death. Waterhemp density and dry biomass were determined by counting and cutting waterhemp at the soil surface from two 0.25 $\mathrm{m}^{2}$ quadrats per plot, placing them in paper bag, drying the biomass to a constant mass at $60 \mathrm{C}$, and then weighing. Corn was harvested from two rows in each plot at maturity with a small plot combine; grain weight and moisture content were recorded. Corn grain yield was expressed as tonnes $\mathrm{ha}^{-1}$ and adjusted to $15.5 \%$ moisture before statistical analysis.

\subsection{Study 2}

Materials and methods were similar to Study 1, with the exception that the weed species evaluated was GR Canada fleabane and present at different sites located near Dresden, Thamseville, Harrow, Ridegtown and Zone Centre; all sites were managed no-till. Table 2 lists GR Canada fleabane sites, planting and herbicide application dates.

\subsection{Statistical Analysis}

For both studies, data were analyzed as a 2 by 5 factorial, variance analysis was performed using PROC GLIMMIX in SAS v. 9.4 (SAS Institute, Cary, NC). Data were pooled across years and locations for analysis. Each location-year indicates an environment for a total of 6 environments in study 1 and 5 environments in study 2. Variances were partitioned into random and fixed effects. Random effects were environment, replication within environment, environment and its interaction with each fixed effect. Fixed effects were Factor One, Factor Two and the interaction between Factor One and Two. The significance of fixed effects was tested using F-tests and random effects were tested using Z-tests with $\alpha$ 
Table 2. Year, location, planting and herbicide application dates for six trials on control of glyphosate-resistant Canada fleabane with Roundup WeatherMAX ${ }^{\circledast}$ plus tolpyralate plus atrazine plus adjuvants, applied postemergence, in Ontario in 2018 and 2019.

\begin{tabular}{cccccc}
\hline Species & Year & Nearest Town & Planting Date & $\begin{array}{c}\text { Emergence } \\
\text { Date }\end{array}$ & $\begin{array}{c}\text { Herbicide } \\
\text { Application Date }\end{array}$ \\
\hline GR horseweed & 2018 & Ridgetown & May 9 & May 20 & May 11 \\
& 2018 & Thamesville & May 9 & May 20 & May 11 \\
& 2018 & Harrow & May 25 & May 31 & May 25 \\
& 2019 & Zone Centre & May 22 & May 29 & May 24 \\
& 2019 & Ridgetown & May 24 & May 30 & May 24 \\
\hline
\end{tabular}

assigned as 0.05 . The assumptions of variance analyses (residuals have mean of zero, are homogeneous, and normally distributed) were tested using a Shapiro-Wilk test of normality and scatterplot of studentized residuals. An appropriate distribution and link were assigned to each parameter to meet those assumptions. Weed control data were transformed using arcsine square root; weed density and biomass data were log-transformed. Treatment comparisons for main effects were performed only when the interaction was not significant. Simple effects were analyzed when the interaction was significant. Treatment comparisons were made based on least-square means using Tukey-Kramer's multiple range test and letter codes were assigned to illustrate statistically significant differences. The means of transformed data were converted back to the original scale for presentation of results.

\section{Results}

\subsection{Study 1}

Data analysis indicated that the Roundup Weather $\mathrm{MAX}^{\oplus}$ by tolpyralate + atrazine interaction was not significant for GR waterhemp density $(P=0.6348)$ and biomass $(P=0.9906)$ and corn yield $(P=0.3225)$ (Table 3$)$. Averaged across all tolpyralate + atrazine treatments, the addition of Roundup WeatherMAX was not significant for GR waterhemp density and biomass and corn yield (Table 3). Averaged across the 0 and $900 \mathrm{~g} \cdot a \mathrm{a} \cdot \mathrm{ha}^{-1}$ of Roundup WeatherMAX, tolpyralate + atrazine reduced GR waterhemp density and biomass, the decrease in density and biomass were greater with the addition of MSO or MSO + UAN compared to UAN. There was no effect of Roundup WeatherMAX ${ }^{\circledR}$ or tolpyralate + atrazine treatment on corn yield (Table 3 ). There was a significant glyphosate by tolpyralate + atrazine interaction for weed control at 2, 4, 8 and 12 WAA $(P<0.0001)($ Table 4$)$.

\subsubsection{Crop Injury}

Corn injury was less than $10 \%$ in all treatments (data not presented).

\subsubsection{Weed Control}

At 2 WAA, tolpyralate + atrazine controlled GR waterhemp 76\%, control increased 
Table 3. Significance of main effects and interactions for glyphosate-resistant waterhemp control, density, and biomass and corn yield with Roundup WeatherMAX ${ }^{\oplus}$ plus tolpyralate plus atrazine from six trials conducted in 2019.

\begin{tabular}{|c|c|c|c|c|c|c|c|}
\hline \multirow[b]{2}{*}{ Main effects } & \multicolumn{4}{|c|}{ Visible Weed Control (\%) } & \multirow[b]{2}{*}{$\begin{array}{l}\text { Density } \\
\left(\text { no } \cdot m^{-2}\right)\end{array}$} & \multirow[b]{2}{*}{$\begin{array}{c}\text { Biomass }^{\mathrm{f}} \\
\left(\mathrm{g} \cdot \mathrm{m}^{-2}\right)\end{array}$} & \multirow[b]{2}{*}{$\begin{array}{c}\text { Yield } \\
\left(\mathrm{t} \cdot \mathrm{ha}^{-1}\right)\end{array}$} \\
\hline & $2 \mathrm{WAA}^{\mathrm{a}}$ & 4 WAA & 8 WAA & 12 WAA & & & \\
\hline \multicolumn{8}{|l|}{ Roundup WeatherMax ${ }^{\circledast}\left(\mathrm{g} \cdot \mathrm{ae} \cdot \mathrm{ha}^{-1}\right)$} \\
\hline 0 & 70 & 74 & 77 & 76 & $161 a$ & $58 a$ & $12.4 a$ \\
\hline 900 & 78 & 81 & 83 & 83 & $156 a$ & $25 a$ & $12.7 a$ \\
\hline $\mathrm{SE}^{\mathrm{b}}$ & 2 & 2 & 2 & 2 & 11 & 4 & 0.2 \\
\hline Roundup Weather $\mathrm{MAX}^{\circledast} \mathrm{P}$-value & 0.0060 & 0.0025 & 0.0068 & 0.0113 & 0.9804 & 0.1193 & 0.5018 \\
\hline \multicolumn{8}{|l|}{ Tolpyralate + atrazine treatments ${ }^{\mathcal{c}}$} \\
\hline No tank mix partner & 3 & 2 & 2 & 3 & $199 a$ & $132 a$ & $11.9 a$ \\
\hline Tolpyralate + Atrazine & 79 & 83 & 90 & 91 & $112 b c$ & $28 b$ & $12.9 a$ \\
\hline Tolpyralate + Atrazine $+\mathrm{MSO}^{\mathrm{d}}$ & 95 & 97 & 98 & 97 & $19 c$ & $2 c$ & $12.5 a$ \\
\hline Tolpyralate + Atrazine $+\mathrm{UAN}^{e}$ & 88 & 90 & 93 & 92 & $169 b$ & $24 b$ & $13.0 \mathrm{a}$ \\
\hline Tolpyralate + Atrazine $+\mathrm{MSO}+\mathrm{UAN}$ & 96 & 97 & 98 & 97 & $13 \mathrm{c}$ & $1 c$ & $12.5 a$ \\
\hline $\mathrm{SE}^{\mathrm{b}}$ & 2 & 2 & 2 & 2 & 11 & 4 & 0.2 \\
\hline Tolpyralate + atrazine $\mathrm{P}$-value & $<0.0001$ & $<0.0001$ & $<0.0001$ & $<0.0001$ & $<0.0001$ & $<0.0001$ & 0.1353 \\
\hline \multicolumn{8}{|l|}{ Interaction } \\
\hline Roundup Weather $\mathrm{MAX}^{\otimes *}$ tolpyralate + atrazine P-value & $<0.0001$ & 0.0001 & 0.0216 & 0.0085 & 0.6348 & 0.9906 & 0.3225 \\
\hline
\end{tabular}

${ }^{\mathrm{a}} \mathrm{WAA}$, weeks after treatment application. ${ }^{\mathrm{b}}$ Standard error of the mean. ${ }^{\mathrm{c}}$ Tolpyralate applied at $30 \mathrm{~g} \cdot \mathrm{ai} \cdot \mathrm{ha}{ }^{-1}$; atrazine applied at $560 \mathrm{~g} \cdot \mathrm{ai} \cdot \mathrm{ha}{ }^{-1}$. ${ }^{\mathrm{d}} \mathrm{MSO}, 0.50 \% \mathrm{v} / \mathrm{v}$. ${ }^{e} \mathrm{UAN}, 2.50 \% \mathrm{v} / \mathrm{v}$. ${ }^{\mathrm{f}}$ Density and biomass collected 8 WAA. Means within column followed by the same lowercase letter are not statistically different according to Tukey-Kramer's LSD $(P=0.05)$.

$11 \%$ to $19 \%$ with the addition of the adjuvants MSO, UAN or MSO + UAN (Table 4). Roundup WeatherMAX ${ }^{\circledast}$ applied alone controlled waterhemp $10 \%$. Glyphosate + tolpyralate + atrazine controlled GR waterhemp 82\%, there was no further increase in control with the addition of MSO, UAN or MSO + UAN. The addition of Roundup WeatherMAX ${ }^{\circledast}$ to tolpyralate + atrazine improved GR waterhemp control $6 \%$. In contrast, when tolpyralate + atrazine was applied with either MSO, UAN or MSO + UAN there was no further increase in GR waterhemp control when co-applied with Roundup WeatherMAX ${ }^{\circledR}$.

At 4 WAA, tolpyralate + atrazine controlled GR waterhemp 79\%, the addition of MSO or MSO + UAN improved GR waterhemp control $17 \%$ and $18 \%$, respectively (Table 4). When MSO was the adjuvant, there was no further increase in GR waterhemp control with the addition of UAN. Roundup WeatherMAX ${ }^{\circledast}$ and Roundup WeatherMAX ${ }^{\oplus}+$ tolpyralate + atrazine controlled waterhemp 8 and $89 \%$, respectively. The addition of MSO to Roundup WeatherMAX ${ }^{\circledast}+$ tolpyralate + atrazine increased GR waterhemp control 9\%; there was no improvement in GR waterhemp control when UAN or MSO + UAN were added to Roundup WeatherMAX ${ }^{\oplus}+$ tolpyralate + atrazine. Similar to 2 WAA, the addition of Roundup WeatherMAX ${ }^{\bowtie}$ to tolpyralate + atrazine improved GR 
Table 4. Glyphosate-resistant waterhemp control 2, 4, 8 and 12 WAA with Roundup WeatherMAX ${ }^{\circledast}$ plus tolpyralate plus atrazine from six trials conducted in Ontario in 2018 and 2019.

\begin{tabular}{|c|c|c|c|}
\hline & \multicolumn{2}{|c|}{ Roundup WeatherMAX $\left(\mathrm{g} \cdot \mathrm{ae} \cdot \mathrm{ha}^{-1}\right)$} & \multirow{2}{*}{ SE } \\
\hline & 0 & 900 & \\
\hline Visible Weed Control2 WAA $(\%)$ & \multicolumn{2}{|c|}{----------------(\%)------------- } & \\
\hline No tank mix partner & $0 c \mathrm{Y}$ & $10 b \mathrm{Z}$ & 1 \\
\hline Tolpyralate + Atrazine ${ }^{c}$ & $76 b \mathrm{Y}$ & $82 a \mathrm{Z}$ & 3 \\
\hline Tolpyralate + Atrazine $+\mathrm{MSO}^{\mathrm{d}}$ & $95 a Z$ & $95 a \mathrm{Z}$ & 1 \\
\hline Tolpyralate + Atrazine $+\mathrm{UAN}^{\mathrm{e}}$ & $87 a \mathrm{Z}$ & $90 a \mathrm{Z}$ & 3 \\
\hline Tolpyralate + Atrazine $+\mathrm{MSO}+\mathrm{UAN}$ & $95 a \mathrm{Z}$ & $96 a \mathrm{Z}$ & 1 \\
\hline $\mathrm{SE}^{\mathrm{b}}$ & 3 & 3 & \\
\hline \multicolumn{4}{|l|}{ Visible Weed Control 4 WAA (\%) } \\
\hline No tank mix partner & $0 c \mathrm{Y}$ & $8 c \mathrm{Z}$ & 1 \\
\hline Tolpyralate + Atrazine & $79 b \mathrm{Y}$ & $89 \mathrm{~b} \mathrm{Z}$ & 3 \\
\hline Tolpyralate + Atrazine $+\mathrm{MSO}$ & $96 a \mathrm{Z}$ & $98 a \mathrm{Z}$ & 1 \\
\hline Tolpyralate + Atrazine + UAN & $88 a b \mathrm{Z}$ & $92 a b \mathrm{Z}$ & 2 \\
\hline Tolpyralate + Atrazine $+\mathrm{MSO}+\mathrm{UAN}$ & $97 a Z$ & $97 a b \mathrm{Z}$ & 1 \\
\hline SE & 3 & 3 & \\
\hline \multicolumn{4}{|l|}{ Visible Weed Control 8 WAA (\%) } \\
\hline No tank mix partner & $0 c \mathrm{Y}$ & $9 b \mathrm{Z}$ & 2 \\
\hline Tolpyralate + Atrazine & $89 a \mathrm{Z}$ & $91 a \mathrm{Z}$ & 3 \\
\hline Tolpyralate + Atrazine + MSO & $97 a \mathrm{Z}$ & $98 a \mathrm{Z}$ & 1 \\
\hline Tolpyralate + Atrazine + UAN & $92 a \mathrm{Z}$ & $93 a \mathrm{Z}$ & 2 \\
\hline Tolpyralate + Atrazine $+\mathrm{MSO}+\mathrm{UAN}$ & $98 a \mathrm{Z}$ & $98 a \mathrm{Z}$ & 1 \\
\hline SE & 3 & 3 & \\
\hline \multicolumn{4}{|l|}{ Visible Weed Control 12 WAA (\%) } \\
\hline No tank mix partner & $0 b \mathrm{Y}$ & $12 b \mathrm{Z}$ & 1 \\
\hline Tolpyralate + Atrazine & $89 a \mathrm{Z}$ & $93 a \mathrm{Z}$ & 3 \\
\hline Tolpyralate + Atrazine $+\mathrm{MSO}$ & $96 a Z$ & $97 a \mathrm{Z}$ & 1 \\
\hline Tolpyralate + Atrazine + UAN & $92 a Z$ & $92 a \mathrm{Z}$ & 2 \\
\hline Tolpyralate + Atrazine + MSO + UAN & $96 a \mathrm{Z}$ & $97 a \mathrm{Z}$ & 1 \\
\hline SE & 4 & 3 & \\
\hline
\end{tabular}

${ }^{a}$ WAA, weeks after treatment application. ${ }^{\mathrm{b}}$ Standard error of the mean. ${ }^{\circ}$ Tolpyralate applied at $30 \mathrm{~g} \cdot \mathrm{ai} \cdot \mathrm{ha} \mathrm{a}^{-1}$; atrazine applied at $560 \mathrm{~g} \cdot \mathrm{ai} \cdot \mathrm{ha} \mathrm{a}^{-1} .{ }^{\mathrm{d}} \mathrm{MSO}, 0.50 \% \mathrm{v} / \mathrm{v}$. ${ }^{\mathrm{e}} \mathrm{UAN}, 2.50 \% \mathrm{v} / \mathrm{v}$. Means within column followed by the same lowercase letter, or means within row followed by the same uppercase letter, are not statistically different according to Tukey-Kramer's LSD $(P=0.05)$.

waterhemp control 10\%; however, there was no increase in GR waterhemp control when Roundup WeatherMAX ${ }^{\circledR}$ was added to tolpyralate + atrazine when applied with MSO, UAN or MSO + UAN. 
At 8 and 12 WAA, tolpyralate + atrazine controlled GR waterhemp 89\%, there was no increase in GR waterhemp control with the addition of MSO, UAN or MSO + UAN (Table 4). At 8 and 12 WAA, Roundup WeatherMAX ${ }^{\circledast}+$ tolpyra- $^{-}$ late + atrazine controlled GR waterhemp 91\% and 93\%, respectively; there was no increase in GR waterhemp control with the addition of MSO, UAN or MSO + UAN.

\subsection{Study 2}

Based on data analysis, the Roundup WeatherMAX ${ }^{\circledast}$ by tolpyralate + atrazine interaction was not significant for GR Canada fleabane density $(P=0.7426)$ or biomass $(P=0.4563)$ (Table 5$)$. Averaged over all tolpyralate + atrazine treatments, the addition of Roundup WeatherMAX ${ }^{\oplus}$ had no effect on GR Canada fleabane biomass or density (Table 5). Averaged over 0 and $900 \mathrm{~g} \cdot \mathrm{ae} \cdot \mathrm{ha}^{-1}$ of Roundup WeatherMAX ${ }^{\oplus}$, tolpyralate + atrazine reduced GR Canada fleabane density; there was no further decrease in density with the addition of MSO, UAN or MSO + UAN. Tolpyralate + atrazine reduced GR Canada fleabane biomass, there was no further decrease in biomass with the addition of MSO, UAN or $\mathrm{MSO}+\mathrm{UAN}$. There was a significant Roundup WeatherMAX ${ }^{\circledR}$ by tolpyralate + atrazine interaction for weed control at 2,4 and 8 WAA $(P<0.0045)$ and corn yield $(P=0.0003)$ (Table 6).

Table 5. Significance of main effects and interactions for glyphosate-resistant Canada fleabane control, density, and biomass and corn yield with Roundup WeatherMAX ${ }^{\circledast}$ plus tolpyralate plus atrazine from six trials conducted in Ontario in 2018 and 2019.

\begin{tabular}{|c|c|c|c|c|c|c|}
\hline \multicolumn{7}{|c|}{ Visible Weed Control (\%) } \\
\hline Main effects & $2 \mathrm{WAA}^{\mathrm{a}}$ & $4 \mathrm{WAA}$ & $8 \mathrm{WAA}$ & $\begin{array}{l}\text { Density }{ }^{\mathrm{f}} \\
\left(\mathrm{no} \cdot \mathrm{m}^{-2}\right)\end{array}$ & $\begin{array}{c}\text { Biomass }^{\mathrm{f}} \\
\left(\mathrm{g} \cdot \mathrm{m}^{-2}\right)\end{array}$ & $\begin{array}{c}\text { Yield } \\
\left(\mathrm{t} \cdot \mathrm{ha}^{-1}\right)\end{array}$ \\
\hline \multicolumn{7}{|l|}{ Roundup WeatherMAX ${ }^{\otimes}\left(\mathrm{g} \cdot \mathrm{ae} \cdot \mathrm{ha}^{-1}\right)$} \\
\hline 0 & 59 & 64 & 71 & $356 a$ & $217 a$ & 11.8 \\
\hline 900 & 74 & 77 & 82 & $497 a$ & $129 a$ & 12.7 \\
\hline $\mathrm{SE}^{\mathrm{b}}$ & 2 & 2 & 2 & 9 & 7 & 0.3 \\
\hline Roundup WeatherMAX ${ }^{\circledast} \mathrm{P}$-value & 0.0272 & 0.0211 & 0.0318 & 0.7579 & 0.2678 & 0.1053 \\
\hline \multicolumn{7}{|l|}{ Tolpyralate + atrazine treatments ${ }^{c}$} \\
\hline No tank mix partner & 4 & 5 & 7 & $575 a$ & $368 a$ & 9.1 \\
\hline Tolpyralate + Atrazine & 68 & 77 & 86 & $174 b$ & $92 b$ & 12.8 \\
\hline Tolpyralate + Atrazine $+\mathrm{UAN}^{\mathrm{e}}$ & 71 & 75 & 83 & $320 b$ & $1188 a b$ & 13.2 \\
\hline Tolpyralate + Atrazine + MSO + UAN & 91 & 92 & 94 & $144 b$ & $91 b$ & 13.3 \\
\hline $\mathrm{SE}^{\mathrm{b}}$ & 2 & 2 & 2 & 9 & 7 & 0.3 \\
\hline Tolpyralate + atrazine P-value & $<0.0001$ & $<0.0001$ & $<0.0001$ & 0.0017 & 0.0017 & 0.0017 \\
\hline \multicolumn{7}{|l|}{ Interaction } \\
\hline Roundup WeatherMAX ${ }^{\oplus *}$ tolpyralate + atrazine P-value & 0.0040 & 0.0045 & 0.0018 & 0.7426 & 0.4563 & 0.0003 \\
\hline
\end{tabular}


Table 6. Glyphosate-resistant Canada fleabane control 2, 4, and 8 WAA and corn yield with glyphosate plus tolpyralate plus atrazine from six trials conducted in Ontario in 2018 and 2019.

\begin{tabular}{|c|c|c|c|}
\hline & \multicolumn{2}{|c|}{ Roundup WeatherMAX ${ }^{\infty}\left(\mathrm{g} \cdot \mathrm{ae} \cdot \mathrm{ha}^{-1}\right)$} & \multirow[b]{2}{*}{ SE } \\
\hline & 0 & 900 & \\
\hline \multicolumn{4}{|l|}{ Visible Weed Control 2 WAA $^{\mathrm{a}}(\%)$} \\
\hline No tank mix partner & $0 d \mathrm{Y}$ & $15 c \mathrm{Z}$ & 2 \\
\hline Tolpyralate + Atrazine $^{c}$ & $58 c Y$ & $78 \mathrm{~b} \mathrm{Z}$ & 4 \\
\hline Tolpyralate + Atrazine $+\mathrm{MSO}^{\mathrm{d}}$ & $94 a \mathrm{Z}$ & $96 a \mathrm{Z}$ & 1 \\
\hline Tolpyralate + Atrazine $+\mathrm{UAN}^{\mathrm{e}}$ & $64 b c \mathrm{Z}$ & $76 \mathrm{~b} \mathrm{Z}$ & 4 \\
\hline Tolpyralate + Atrazine $+\mathrm{MSO}+\mathrm{UAN}$ & $92 a b Z$ & $91 a b \mathrm{Z}$ & 2 \\
\hline $\mathrm{SE}^{\mathrm{b}}$ & 3 & 3 & \\
\hline \multicolumn{4}{|l|}{ Visible Weed Control4 WAA (\%) } \\
\hline No tank mix partner & $0 d \mathrm{Y}$ & $18 \mathrm{c} \mathrm{Z}$ & 3 \\
\hline Tolpyralate + Atrazine & $68 c \mathrm{Y}$ & $86 \mathrm{ab} Z$ & 4 \\
\hline Tolpyralate + Atrazine + MSO & $96 a \mathrm{Z}$ & $96 a \mathrm{Z}$ & 2 \\
\hline Tolpyralate + Atrazine + UAN & $70 b c \mathrm{Z}$ & $78 \mathrm{~b} \mathrm{Z}$ & 4 \\
\hline Tolpyralate + Atrazine $+\mathrm{MSO}+\mathrm{UAN}$ & $92 a b \mathrm{Z}$ & $93 a b \mathrm{Z}$ & 2 \\
\hline SE & 4 & 3 & \\
\hline \multicolumn{4}{|l|}{ Visible Weed Control 8 WAA (\%) } \\
\hline No tank mix partner & $0 b \mathrm{Y}$ & $25 b \mathrm{Z}$ & 4 \\
\hline Tolpyralate + Atrazine & $80 a \mathrm{Z}$ & $91 a \mathrm{Z}$ & 3 \\
\hline Tolpyralate + Atrazine + MSO & $97 a \mathrm{Z}$ & $97 a \mathrm{Z}$ & 2 \\
\hline Tolpyralate + Atrazine + UAN & $83 a \mathrm{Z}$ & $84 a \mathrm{Z}$ & 4 \\
\hline Tolpyralate + Atrazine $+\mathrm{MSO}+\mathrm{UAN}$ & $94 a \mathrm{Z}$ & $95 a \mathrm{Z}$ & 2 \\
\hline SE & 4 & 3 & \\
\hline \multicolumn{4}{|l|}{ Yield $\left(\mathrm{t} \cdot \mathrm{ha}^{-1}\right)$} \\
\hline No tank mix partner & $6.9 a \mathrm{Y}$ & $11.3 \mathrm{aZ}$ & 0.8 \\
\hline Tolpyralate + Atrazine & $12.7 \mathrm{~b} \mathrm{Z}$ & 12.9 a Z & 0.3 \\
\hline Tolpyralate + Atrazine + MSO & $12.8 \mathrm{~b} \mathrm{Z}$ & $13.1 \mathrm{aZ}$ & 0.4 \\
\hline Tolpyralate + Atrazine + UAN & $12.9 \mathrm{~b} \mathrm{Z}$ & $13.4 \mathrm{aZ}$ & 0.3 \\
\hline Tolpyralate + Atrazine $+\mathrm{MSO}+\mathrm{UAN}$ & $13.9 b \mathrm{Z}$ & 12.7 a Z & 0.5 \\
\hline SE & 0.4 & 0.3 & \\
\hline
\end{tabular}

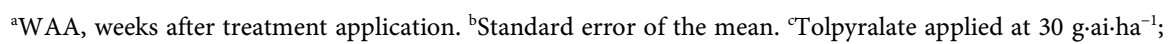
atrazine applied at $560 \mathrm{~g} \cdot \mathrm{ai} \cdot \mathrm{ha} \mathrm{a}^{-1} .{ }^{\mathrm{d}} \mathrm{MSO}, 0.50 \% \mathrm{v} / \mathrm{v}$. ${ }^{\mathrm{e}} \mathrm{UAN}, 2.50 \% \mathrm{v} / \mathrm{v}$. Means within column followed by the same lowercase letter, or means within row followed by the same uppercase letter, are not statistically different according to Tukey-Kramer's LSD $(P=0.05)$.

\subsubsection{Crop Injury}

Crop injury was less than $10 \%$ in all treatments (data not presented).

\subsubsection{Weed Control}

Tolpyralate + atrazine controlled GR Canada fleabane $58 \%$ at 2 WAA; there was no improvement in control with the addition of UAN (Table 6). The addition of 
MSO or MSO + UAN to tolpyralate + atrazine improved GR Canada fleabane control 36\% and 34\%, respectively. Roundup WeatherMAX ${ }^{\circledR}$ applied alone controlled GR Canada fleabane 15\%. Roundup WeatherMAX ${ }^{\circledR}+$ tolpyralate + atrazine controlled GR Canada fleabane 78\%, the addition of MSO improved GR Canada fleabane control 18\%; there was no improvement in GR Canada fleabane control with the addition of UAN or MSO + UAN. When MSO was the adjuvant, there was no further increase in GR Canada fleabane control with the addition of UAN. The addition of Roundup WeatherMAX ${ }^{\oplus}$ to tolpyralate + atrazine improved control of GR Canada fleabane $20 \%$. In contrast, when tolpyralate + atrazine was applied with either MSO, UAN or MSO + UAN there was no further increase in GR Canada fleabane control with the addition of glyphosate.

At 4 WAA, tolpyralate + atrazine controlled GR Canada fleabane $68 \%$. The addition of MSO or MSO + UAN improved control $28 \%$ and $24 \%$, respectively. In contrast, there was no increase in GR Canada fleabane control with the addition of UAN. Roundup WeatherMAX ${ }^{\circledR}$ and Roundup WeatherMAX ${ }^{\circledast}+$ tolpyralate + atrazine controlled GR Canada fleabane $18 \%$ and $86 \%$, respectively. There was no further increase in control when MSO, UAN or MSO + UAN were added. The addition of Roundup WeatherMAX ${ }^{\oplus}$ to tolpyralate + atrazine improved GR Canada fleabane control 18\%; however there was no increase in GR Canada fleabane control when Roundup WeatherMAX ${ }^{\circledR}$ was added to tolpyralate + atrazine when applied with MSO, UAN or MSO + UAN.

At 8 WAA, tolpyralate + atrazine controlled GR Canada fleabane $80 \%$ and there was no increase in GR Canada fleabane control with the addition of MSO, UAN or MSO + UAN (Table 6). Roundup WeatherMAX ${ }^{\oplus}$ and Roundup WeatherMAX ${ }^{\oplus}+$ tolpyralate + atrazine controlled GR Canada fleabane $25 \%$ and $91 \%$, respectively, there was no increase in control with the addition of MSO, UAN or MSO + UAN.

\subsubsection{Yield}

GR Canada fleabane interference reduced corn yield up to 50\% (highest yielding treatment compared to the weedy control). Reduced GR Canada fleabane interference with tolpyralate + atrazine, without and with adjuvants, increased corn yield $84 \%$ to $101 \%$ relative to the weedy control.

\section{Discussion}

POST-applied herbicides must contact the leaf surface of the target species and cross the cuticle and enter the living portion of the plant to be effective. Adjuvants improve herbicide performance by altering application characteristics, physical and/or chemical properties of herbicides [37]. The tolpyralate label recommends the addition of MSO and UAN as adjuvants for enhanced herbicide efficacy [25] [28]. Glyphosate (Roundup WeatherMAX ${ }^{\oplus}$ ) has a proprietary blend of surfactants included in the formulation [26]. The addition of Roundup WeatherMAX ${ }^{\oplus}$ to tolpyralate + atrazine improved the control of GR waterhemp and Canada fleabane at 2 and 4 WAA, in the absence of MSO, UAN or MSO + 
UAN. At 2 and 4 WAA, the addition of Roundup WeatherMAX ${ }^{ø}$ to tolpyralate + atrazine increased GR waterhemp control by $6 \%$ and $10 \%$, and increased GR Canada fleabane control by $20 \%$ and $18 \%$, respectively. Armel et al. [52] also reported improved control of common ragweed and giant foxtail with mesotrione (105 or $140 \mathrm{~g} \cdot \mathrm{ai} \cdot \mathrm{ha}^{-1}$ ) when in a tankmix with glyphosate compared to mesotrione alone. Soltani et al. [34] reported an interaction between glyphosate and 2,4-D where corn injury is enhanced with the addition of glyphosate, speculated to be caused by the aggressive adjuvant system in a commercial glyphosate formulation. In contrast to results from Soltani et al. [34], crop injury was not accentuated with the addition of glyphosate to tolpyralate + atrazine, however there was an influence on weed control. Glyphosate tank mixtures are reported to be complementary for herbicides like saflufenacil, chlorimuron and imazethapyr, exhibiting an additive effect [35] [38]. In contrast, addition of glyphosate to fomesafen and sulfentrazone was antagonistic and reduced absorption and translocation within various weed species [38]. Research by Deen et al. [36] found that quizalofop-p-ethyl (high rate) plus glyphosate and quizalofop-p-ethyl plus the recommended adjuvant provided similar control of GR volunteer corn, suggesting that the adjuvant in the glyphosate formulation could replace the recommended adjuvant on the quizalofop-p-ethyl label. At lower rates of quizalofop-p-ethyl, greatest control was achieved when the recommended adjuvant was used [36].

Removing unnecessary adjuvants from herbicide applications allows farmers to spend less money on their weed control program while still achieving a high level of weed control. In Ontario, adjuvants can cost between $\$ 1.00$ to $10.00 \mathrm{ha}^{-1}$. In this specific example, using both UAN and MSO with tolpyralate would require the farmer to spend over $\$ 10.00 \mathrm{ha}^{-1}$ on the adjuvant system (Agris Co-operative Ltd., personal communication). Results of this research, irrespective of Roundup WeatherMAX ${ }^{\circledR}$ presence, show the greatest control of both species at 2 and 4 WAA was achieved when MSO was added to tolpyralate + atrazine, and there was no increase in weed control with the addition of UAN. The results of this study conclude that there was no increase in control when UAN was added to MSO, therefore the addition of UAN to tolpyralate + atrazine was not necessary for the control of GR waterhemp and GR Canada fleabane. In Ontario, this may allow the farmer to save up to $\$ 2.50 \mathrm{ha}^{-1}$; this could be a substantial savings if used over a large number of hectares.

In the absence of Roundup WeatherMAX ${ }^{\circledR}$, control of GR waterhemp and GR Canada fleabane was affected by adjuvant selection. Without the addition of an adjuvant, tolpyralate + atrazine provided less than $70 \%$ control of Canada fleabane (Table 6) and less than $80 \%$ control of waterhemp (Table 3) at 2 and 4 WAA. Previous research by Dayan et al. [39] found that in the absence of adjuvants, sulfentrazone absorption and phototoxic effects on weeds was reduced, although when included, adjuvants overcame barriers to herbicide absorption in plant leaves. As an activator adjuvant, MSO is an oil adjuvant made from soybean and influences various properties of an herbicide spray droplet leading to 
improved efficacy [40]. Tolpyralate + atrazine + MSO provided the greatest control of GR Canada fleabane at 2 and $4 \mathrm{WAA}$, and waterhemp at $4 \mathrm{WAA}$. The increased herbicidal activity observed with the addition of MSO may be attributed to the greater absorption and retention of the herbicide on plant leaf as a result of various physical and chemical changes to the herbicide solution [31] [41]. Reduced surface tension and contact angle of spray droplets promote the penetration of herbicides through the leaf cuticle [31]. Herbicidal activity of topramezone and isoxaflutole, two other Group 27 herbicides, is also improved with the addition of MSO [31] [41]. Hutchinson et al. [42] found that MSO enhanced activity of metribuzin, resulting in greater control of lambsquarters, hairy nightshade, redroot pigweed and kochia when compared to nonionic surfactants. The addition of UAN to tolpyralate + atrazine with MSO did not increase the control of GR waterhemp and GR Canada fleabane (Table 3 and Table 6). This study concludes that the addition of MSO to tolpyralate + atrazine improves the control of GR waterhemp and GR Canada fleabane, but there is no improvement in weed control when UAN is added to tolpyralate + atrazine. This is consistent with research conducted by Idziak et al. [43] and Young and Hart [41] where no increase in weed control was observed with the addition of UAN to MSO. In contrast, Gronwald et al. 1993 [44] reported an increase in herbicidal activity with the addition of a nitrogen fertilizer due to the influence of ammonium ions on herbicide absorption into the plant. There was no improvement in GR waterhemp and GR Canada fleabane with the addition of an adjuvant at 8 and 12 WAA. This study found that the addition of MSO to tolpyralate + atrazine increases the speed of GR waterhemp and GR Canada fleabane control, but end-of-season weed control is similar. The current tolpyralate label in the US claims partial control of GR Canada fleabane; GR Canada fleabane does not appear on the Canadian label. Results from this study conclude that tolpyralate + atrazine controls GR Canada fleabane [25] [28]. Metzger et al. [27] also found GR Canada fleabane control with tolpyralate + atrazine equivalent to bromoxynil + atrazine and dicamba/atrazine, the current industry standards for control of GR Canada fleabane in corn in Canada. In addition, results of this study are similar to Benoit et al. [8] who reported that POST-applied tolpyralate + atrazine controlled GR waterhemp in corn. Osipitan et al. [45] found that tolpyralate + atrazine controlled waterhemp at lower than the label rate at 60 days after application.

Corn is susceptible to herbicide injury when plants are young and rapidly growing, possessing a thin cuticle that allows for rapid uptake of the herbicide [46]. White bleaching is a characteristic corn injury symptom from HPPD-inhibiting herbicides. Corn injury across all experiments was less than $10 \%$ (data not shown); however, injury symptoms appeared as gray, water-soaked lesions, likely a result of the adjuvants used [47]. The addition of MSO to mesotrione increased herbicide uptake in sorghum resulting in increased levels of crop injury, sorghum outgrew the injury and yield was not impacted [43]. Metzger et al. [47] found tolpyralate + atrazine + MSO + UAN to 
have a wide margin of crop safety on some commonly used corn hybrids in Ontario when used according to label recommendations, although environmental variables may affect corn injury levels. In contrast to research by Soltani et al. [34] that reported increased crop injury when glyphosate was added to 2,4-D, the addition of glyphosate to tolpyralate + atrazine did not enhance crop injury. Any visible crop injury symptoms in these studies did not affect corn yield. Left uncontrolled, GR Canada fleabane reduced corn grain yields by up to $50 \%$ in this study, which is similar to research by Ford et al. [48] that observed a $69 \%$ yield loss due GR Canada fleabane interference in corn.

\section{Conclusion}

Results indicate that the addition of Roundup WeatherMAX enhances herbicidal activity of tolpyralate + atrazine; however, the addition of MSO is still required to enhance the speed of activity. The inclusion of a commercial glyphosate formulation in the tankmix will increase the spectrum of weeds controlled and an additional mode of action will reduce the selection intensity for Group 27 resistant weeds. When Roundup WeatherMAX ${ }^{\circledR}$ is absent, the control of GR waterhemp and GR Canada fleabane was influenced by the adjuvant at 2 and 4 WAA. By 8 WAA, all treatments provided good to excellent control of GR waterhemp and GR Canada fleabane. This study concludes that with tolpyralate + atrazine there is no benefit of adding UAN to MSO for control of GR waterhemp or Canada fleabane. This may allow growers to reduce costs and still achieve excellent control of these species with tolpyralate + atrazine. Group 27-resuistance has been reported in Amaranthus species in the United States [49] [50] [51] [52]. Incorporating multiple effective modes of action, in addition to a diverse crop and herbicide rotation will enhance the long-term effectiveness of this herbicide mode-of-action.

\section{Acknowledgements}

Funding for this project was provided in part by ISK Biosciences Inc. and Grain Farmers of Ontario (GFO).

\section{Conflicts of Interest}

The authors declare no conflicts of interest regarding the publication of this paper.

\section{References}

[1] Schryver, M., Soltani, N., Hooker, D.C., Robinson, D.E., Tranel, P.J. and Sikkema, P.H. (2017) Glyphosate-Resistant Waterhemp (Amaranthus tuberculatus var. rudis) in Ontario, Canada. Canadian Journal of Plant Science, 97, 1057-1067. https://doi.org/10.1139/CJPS-2016-0371

[2] Hartzler, R.G., Battles, B.A. and Nordby, D. (2004) Effect of Common Waterhemp (Amaranthus rudis) Emergence Date on Growth and Fecundity in Soybean. Weed Science, 52, 242-245. https://doi.org/10.1614/WS-03-004R 
[3] Soltani, N., Vyn, J.D. and Sikkema, P.H. (2009) Control of Common Waterhemp (Amaranthus tuberculatus var. rudis) in Corn and Soybean with Sequential Herbicide Applications. Canadian Journal of Plant Science, 89, 127-132. https://doi.org/10.4141/CJPS08051

[4] Buhler, D.D. and Hartzler, R.G. (2001) Emergence and Persistence of Seed of Velvetleaf, Common Waterhemp, Woolly Cupgrass, and Giant Foxtail. Weed Science, 49, 230-235. https://doi.org/10.1614/0043-1745(2001)049[0230:EAPOSO]2.0.CO;2

[5] Steckel, L., Sprague, C., Stoller, E.W. and Simmons, F.W (2007) Tillage, Cropping System, and Soil Depth Effects on Common Waterhemp (Amaranthus rudis) Seed-Bank Persistence. Weed Science, 55, 235-239.

https://doi.org/10.1614/WS-06-198

[6] Costea, M, Weaver, S.E. and Tardif, F.J. (2005) The Biology of Invasive Alien Plants in Canada. 3. Amaranthus tuberculatus (Moq.) Sauer var. Rudis (Sauer) Costea \& Tardif. Canadian Journal of Plant Science, 85, 507-522. https://doi.org/10.4141/P04-101

[7] Heap, I. (2019) Retrieved from The International Survey of Herbicide Resistant Weeds. http://www.psu.edu/

[8] Benoit, L., Soltani, N., Hooker, D.C., Robinson, D.E. and Sikkema, P.H. (2019) Efficacy of HPPD-Inhibiting Herbicides Applied Preemergence or Postemergence for Control of Multiple Herbicide Resistant Waterhemp [Amaranthus tuberculatus (Moq.) Sauer]. Canadian Journal of Plant Science, 99, 379-383. https://doi.org/10.1139/cjps-2018-0320

[9] Nandula, V.K., Ray, J.D., Ribeiro, D.N., Pan, Z. and Reddy, K.N. (2013) Glyphosate Resistance in Tall Waterhemp (Amaranthus tuberculatus) from Mississippi Is Due to Both Altered Target-Site and Nontarget-Site Mechanisms. Weed Science, 61, 374-383. https://doi.org/10.1614/WS-D-12-00155.1

[10] Chatham, L.A., Wu, C., Riggins, C.W., Hager, A.G., Young B.G., Roskamp, G.K. and Tranel, P.J. (2015) EPSPS Gene Amplification Is Present in the Majority of Glyphosate-Resistant Illinois Waterhemp (Amaranthus tuberculatus) Populations. Weed Technology, 29, 48-55. https://doi.org/10.1614/WT-D-14-00064.1

[11] Bensch, C., Horak, M. and Peterson, D. (2003) Interference of Redroot Pigweed (Amaranthus retroflexus), Palmer Amaranth (A. palmeri), and Common Waterhemp (A. rudis) in Soybean. Weed Science, 51, 37-43. https://doi.org/10.1614/0043-1745(2003)051[0037:IORPAR]2.0.CO;2

[12] Weaver, S.E. (2001) The Biology of Canadian Weeds. 115. Conyza canadensis. Canadian Journal of Plant Science, 81, 867-875. https://doi.org/10.4141/P00-196

[13] Buhler, D.D. and Owen, M.D. (1997) Emergence and Survival of Horseweed (Conyza canadensis). Weed Science, 45, 98-101. https://doi.org/10.1017/S0043174500092535

[14] Tozzi, E., Beckie, H., Weiss, R., Gonzalez-Andujar, J.L., Storkey, J., Cici, S.Z. and Van Acker, R.C. (2014) Seed Germination Response to Temperature for a Range of International Populations of Conyza canadensis. Weed Resin, 54, 178-185. https://doi.org/10.1111/wre.12065

[15] Regehr, D.L. and Bazzaz, F.A. (1979) The Population Dynamics of Erigeron canadensis, a Successional Winter Annual. Journal of Ecology, 67, 923-933. https://doi.org/10.2307/2259221

[16] Shields, E.J., Dauer, J.T., VanGessel, M.J. and Neumann, G (2006) Horseweed (Conyza canadensis) Seed Collected in the Planetary Boundary Layer. Weed Sci- 
ence, 54, 1063-1067. https://doi.org/10.1614/WS-06-097R1.1

[17] Nandula, V., Eubank, T., Poston, D., Koger, C. and Reddy, K. (2006) Factors Affecting Germination of Horseweed (Conyza canadensis). Weed Science, 54, 898-902. https://doi.org/10.1614/WS-06-006R2.1

[18] VanGessel, M. (2001) Glyphosate-Resistant Horseweed from Delaware. Weed Science, 49, 703-705. https://doi.org/10.1614/0043-1745(2001)049[0703:RPRHFD]2.0.CO;2

[19] Budd, C.M., Soltani, N., Robinson, D.E., Hooker, D.C., Miller, R.T. and Sikkema, P.H. (2018) Distribution of Glyphosate and Cloransulam-Methyl Resistant Canada Fleabane [Conyza canadensis (L.) Cronq.] in Ontario. Canadian Journal of Plant Science, 98, 492-497. https://doi.org/10.1139/CJPS-2016-0346

[20] Ge, X., d'Avignon, D.A., Ackerman, J.J. and Sammons, R.D. (2010) Rapid Vacuolar Sequestration: The Horseweed Glyphosate Resistance Mechanism. Pest Management Science, 66, 345-348. https://doi.org/10.1002/ps.1911

[21] González-Torralva, F., Rojano-Delgado, A.M., de Castro, M.D.L., Mülleder, N. and De Prado, R. (2012) Two Non-Target Mechanisms Are Involved in Glyphosate-Resistant Horseweed (Conyza canadensis L. Cronq.) Biotypes. Journal of Plant Physiology, 169, 1673-1679. https://doi.org/10.1016/j.jplph.2012.06.014

[22] Page, E., Grainger, C., Laforest, M., Nurse, R., Rajcan, I., Bae, J. and Tardif, F.J. (2018) Target and Non-Target Site Mechanisms Confer Resistance to Glyphosate in Canadian Accessions of Conyza canadensis. Weed Science, 66, 234-245. https://doi.org/10.1017/wsc.2017.69

[23] Metzger, B.A., Soltani, N., Raeder, A.J., Hooker, D.C., Robinson, D.E. and Sikkema, P.H. (2018) Tolpyralate Efficacy: Part 2. Comparison of Three Group 27 Herbicides Applied POST for Annual Grass and Broadleaf Weed Control in Corn. Weed Technology, 32, 707-713. https://doi.org/10.1017/wet.2018.81

[24] Metzger, B.A., Soltani, N., Raeder, A.J., Hooker, D.C., Robinson, D.E. and Sikkema, P.H. (2018) Tolpyralate Efficacy: Part 1. Biologically Effective Dose of Tolpyralate for Control of Annual Grass and Broadleaf Weeds in Corn. Weed Technology, 32, 698-706. https://doi.org/10.1017/wet.2018.82

[25] Anonymous (2017) SHIELDEX ${ }^{\circledast}$ 400SC Herbicide Label. SummitAgro. ISK Biosciences Corporation, Durham, NC.

[26] Anonymous (2019) Roundup WeatherMAX ${ }^{\bowtie}$ With Transorb 2 Technology. Monsanto Canada ULC, Winnipeg, Manitoba, Canada.

[27] Metzger, B.A., Soltani, N., Raeder, A.J., Hooker, D.C., Robinson, D.E. and Sikkema, P.H. (2019) Multiple Herbicide-Resistant Horseweed (Conyza canadensis) Dose Response to Tolpyralate and Tolpyralate plus Atrazine and Comparison to Industry Standard Herbicides in Corn. Weed Technology, 33, 366-373.

https://doi.org/10.1017/wet.2019.20

[28] Anonymous (2019) SHIELDEX ${ }^{\circledR}$ 400SC Herbicide Label. ISK Biosciences Corporation, Concord, $\mathrm{OH}$.

[29] Xu, L., Zhu, H., Ozkan, H.E., Bagley, W.E. and Krause, C.R. (2011) Droplet Evaporation and Spread on Waxy and Hairy Leaves Associated with Type and Concentration of Adjuvants. Pest Management Science, 67, 842-851. https://doi.org/10.1002/ps.2122

[30] Xu, L.Y., Zhu, H.P., Ozkan, H.E., Bagley, W.E., Derksen, R.C. and Krause, C.R. (2010) Adjuvant Effects on Evaporation Time and Wetted Area of Droplets on Waxy Leaves. American Society of Agricultural and Biological Engineers, 51, 13-20. https://doi.org/10.13031/2013.29495 
[31] Zhang, J., Jaeck, O., Menegat, A., Zhang, Z., Gerhards, R. and Ni, H. (2013) The Mechanism of Methylated Seed Oil on Enhancing Biological Efficacy of Topramezone on Weeds. PLOS ONE, 8, e74280. https://doi.org/10.1371/journal.pone.0074280

[32] Woznica, Z., Nalewaja, J.D., Messersmith, C.G. and Milkowski, P. (2003) Quinclorac Efficacy as Affected by Adjuvants and Spray Carrier Water. Weed Technology, 17, 582-588. https://doi.org/10.1614/0890-037X(2003)017[0582:QEAABA]2.0.CO;2

[33] Bayer (2019) Roundup. https://www.roundup.ca/en/the-roundup-advantage

[34] Soltani, N., Shropshire, C. and Sikkema, P.H. (2018) Tank Mixture of Glyphosate with 2, 4-D Accentuates 2, 4-D Injury in Glyphosate-Resistant Corn. Canadian Journal of Plant Science, 98, 889-896. https://doi.org/10.1139/cjps-2017-0241

[35] Eubank, T.W., Nandula, V.K., Reddy, K.N., Poston, D.H. and Shaw, D.R. (2013) Saflufenacil Efficacy on Horseweed and Its Interaction with Glyphosate. Weed Biology and Management, 13, 135-143. https://doi.org/10.1111/wbm.12022

[36] Deen, W., Hamill, A., Shropshire, C., Soltani, N. and Sikkema, P.H. (2006) Control of Volunteer Glyphosate-Resistant Corn (Zea mays) in Glyphosate-Resistant Soybean (Glycine max). Weed Technology, 20, 261-266.

https://doi.org/10.1614/WT-02-128.1

[37] Curran, W.S., McGlamery, M.D., Liebl, R.A. and Lingenfelter, D.D. (1999) Adjuvants for Enhancing Herbicide. Penn State Extension. Pennsylvania State University, State College, PA. http://www.psu.edu/

[38] Starke, R.J. and Oliver, L.R. (1998) Interaction of Glyphosate with Chlorimuron, Fomesafen, Imazethapyr, and Sulfentrazone. Weed Science, 46, 652-660. https://doi.org/10.1017/S0043174500089670

[39] Dayan, F.E., Green, H.M., Weete, J.D. and Hancock, H.G. (1996) Postemergence Activity of Sulfentrazone: Effects of Surfactants and Leaf Surfaces. Weed Science, 44, 797-803. https://doi.org/10.1017/S0043174500094741

[40] Penner, D. (2000) Activator Adjuvants. Weed Technology, 14, 785-791. https://doi.org/10.1614/0890-037X(2000)014[0785:AA]2.0.CO;2

[41] Young, B.G. and Hart, S.E. (1998) Optimizing Foliar Activity of Isoxaflutole on Giant Foxtail (Setaria faberi) with Various Adjuvants. Weed Science, 46, 397-402. https://doi.org/10.1017/S0043174500090792

[42] Hutchinson, P.J., Eberlein, C.V. and Tonks, D.J. (2004) Broadleaf Weed Control and Potato Crop Safety with Postemergence Rimsulfuron, Metribuzin, and Adjuvant Combinations. Weed Technology, 18, 750-756. https://doi.org/10.1614/WT-03-172R1

[43] Idziak, R., Skrzypczak, W., Waligora, H. and Woznica, Z. (2013) The Effect of Mesotrione Applied with Adjuvants on Weed Control Efficacy and Forage Sorghum Tolerance. Turkish Journal of Agriculture and Forestry, 37, 265-270.

[44] Gronwald, J.W., Jourdan, S.W., Wyse, D.L., Somers, D.A. and Magnusson, M.U. (1993) Effect of Ammonium Sulfate on Absorption of Imazethapyr by Quackgrass (Elytrigia repens) and Maize (Zea mays) Cell Suspension Cultures. Weed Science, 41, 325-334. https://doi.org/10.1017/S0043174500052012

[45] Osipitan, O.A., Scott, J.E. and Knezevic, S.Z. (2018) Tolpyralate Applied alone and with Atrazine for Weed Control in Corn. The Journal of Agricultural Science, 10 , 32-39. https://doi.org/10.5539/jas.v10n10p32

[46] Currier, H.B. and Dybing, C.D. (1959) Foliar Penetration of Herbicides-Review 
and Present Status. Weeds, 7, 195-213. https://doi.org/10.2307/4040221

[47] Metzger, B.A., Soltani, N., Raeder, A.J., Hooker, D.C., Robinson, D.E. and Sikkema, P.H. (2019) Effect of Hybrid Varieties, Application Timing, and Herbicide Rate on Field Corn Tolerance to Tolpyralate plus Atrazine. Weed Science, 67, 475-484. https://doi.org/10.1017/wsc.2019.34

[48] Ford, L., Soltani, N., Robinson, D.E., Nurse, R.E., McFadden, A. and Sikkema, P.H. (2014) Canada Fleabane (Conyza canadensis) Control with Preplant Applied Residual Herbicides Followed by 2, 4-D Choline/Glyphosate DMA Applied Postemergence in Corn. Canadian Journal of Plant Science, 94, 1231-1237. https://doi.org/10.4141/cjps2013-403

[49] Jhala, A.J., Sandell, L.D., Rana, N., Kruger, G.R. and Knezevic, S.Z. (2014) Confirmation and Control of Triazine and 4-Hydroxyphenylpyruvate Dioxygenase-Inhibiting Herbicide-Resistant Palmer Amaranth (Amaranthus palmeri) in Nebraska. Weed Technology, 28, 28-38. https://doi.org/10.1614/WT-D-13-00090.1

[50] Kohrt, J.R. and Sprague, C.L. (2017) Response of a Multiple-Resistant Palmer Amaranth (Amaranthus palmeri) Population to Four HPPD-Inhibiting Herbicides Applied alone and with Atrazine. Weed Science, 65, 534-545. https://doi.org/10.1017/wsc.2017.28

[51] Hausman, N.E., Singh, S., Tranel, P.J., Riechers, D.E., Kaundun, S.S., Polge, N.D., Thomas, D.A. and Hager, A.G. (2011) Resistance to HPPD-Inhibiting Herbicides in a Population of Waterhemp (Amaranthus tuberculatus) from Illinois, United States. Pest Management Science, 67, 258-261. https://doi.org/10.1002/ps.2100

[52] Armel, G.R., Wilson, H.P., Richardson, R.J. and Hines, T.E. (2003) Mesotrione alone and in Mixtures with Glyphosate in Glyphosate-Resistant Corn (Zea mays). Weed Technology, 17, 680-685. https://doi.org/10.1614/WT02-77 